\title{
Wir haben auch 2008 viel vor Ein Blick nach vorn
}

\begin{abstract}
Auch im neuen Jahr hat die Arbeitsgemeinschaft für nephrologisches Personal wieder viel vor: Neben dem Pflegetag, der im Rahmen des Nephrologiekongresses im September in Tübingen stattfinden wird, sind zahlreichen Fort- und Weiterbildungen geplant, der AfnP beteiligt sich am „Bündnis Niere“, an der Überarbeitung der Leitlinie für angewandte Hygiene in Dialyseeinrichtung und baut das Angebot für Techniker in Dialyseeinrichtungen aus. Last but not least findet am 25. und 26. Oktober zum 30. Mal das AfnP-Symposium statt. Hier wird erneut der Nephrologische Pflegepreis vergeben.
\end{abstract}

Die Arbeitsgemeinschaft für nephrologisches Personal e. V. (AfnP) ist der größte nationale, gemeinnützige $\mathrm{Be}$ rufsverband für alle Berufsgruppen aus dem Tätigkeitsfeld Nephrologie, Dialyse und Transplantationsmedizin. Alle Berufsgruppen sind in der AfnP herzlich willkommen. Die Mitglieder der AfnP leben im gesamten deutschsprachigen Raum (Deutschland, Österreich, Schweiz), aber auch in Frankreich, Spanien, Belgien und Luxemburg.

\section{Pflegetag im Rahmen des Nephro- logiekongresses}

Dank der beständigen Zunahme an Mitgliedern haben wir nun bald 1500 Mitglieder. Die AfnP ist ein starker Berufsverband, der für die nephrologische Pflege in den letzten 30 Jahren der Verbandsgeschichte vieles erreicht hat und in der Zukunft noch vieles erreichen will. Ein Hauptanliegen ist es, alle nephrologisch tätigen Berufsgruppen zu ver- knüpfen, um dadurch den Austausch von Ideen und die Basis für gemeinsame Projekte zu schaffen.

Um diese Kooperationen und den Ausbau der Kontakte zu allen anderen im Bereich der Nephrologie tätigen Fachverbände sind wir kontinuierlich bemüht. Gemeinsam mit den beiden Ärzteverbänden GfN (Gesellschaft für Nephrologie) und DAGKN (Deutsche Arbeitsgemeinschaft für Klinische Nephrologie) sowie dem Pflegeverband der Transplantationskräfte AKTX e. V. und dem Fachverband nephrologischer Berufsgruppen (fnb) gestalten wir den diesjährigen Pflegetag am 27. September 2008 in Tübingen, der im Rahmen des jährlichen Kongresses für Nephrologie stattfindet.

Das Vormittagsprogramm beinhaltet Vorträge aus den Bereichen Hygiene, Gefäßzugänge, Peritonealdialyse, Antikoagulation und Lebendspende. Am Nachmittag hat man die Qual der Wahl und muss sich zwischen fünf verschiedenen Workshops entscheiden. Das ausführliche Programm und alles Weitere finden Sie auf unserer Homepage www.afnp.de.

\section{In den verschiedensten Bereichen aktiv}

Wo immer es im Bereich der Nephrologie und der nephrologischen Pflege etwas zu tun gibt ist, will die AfnP aktiv mitarbeiten. Die zweite Auflage der Leitlinie für angewandte Hygiene in Dialyseeinrichtung wird teilweise umgearbeitet und ergänzt. Im Bereich der Fachweiterbildung Nephrologie wird der derzeitige Lehrplan an die aktuelle Ausbildungsverordnung für Gesundheitspfleger und Gesundheitspflegerinnen angepasst. Dafür werden Handlungsfelder/Lernfelder erstellt.

Das „Bündnis Niere“, dem sich alle in der Nephrologie beteiligten Gruppen (Anbieter von Dialyseprodukten, Pflege-, Ärzte- und Patientenverbände) angeschlossen haben, erstellt ein Positionspapier und erörtert Möglichkeiten, die Zukunft und Qualität der Dialysebehandlung zu sichern.

Für die Techniker in Dialyseeinrichtungen haben wir eine eigene Arbeitsgruppe eingerichtet, die in diesem Jahr für den technischen Teil des Programms beim 30. AfnP-Symposium am 25. und 26. Oktober 2008 zuständig ist.

\section{Qualität der nephrologischen Pflege verbessern}

Auch in diesem Jahr wird die AfnP zusammen mit der Dialyse aktuell den För- 
derpreis Nephrologische Pflege vergeben. Das Preisgeld von 1500 Euro wird vom Georg Thieme Verlag gestiftet. Einreichen können Sie alle Arbeiten über praxisrelevante Projekte, die Sie in Ihrem beruflichen Umfeld umgesetzt haben und mit denen Sie bereits erste Erfahrungen im Alltag sammeln konnten. Das Themenspektrum ist sehr weit gefasst und umfasst alle Aspekte rund um die Nierenersatztherapie.

Bis zum 31. Juli kann sich jeder um den Pflegepreis bewerben, der in der Nephrologie tätig ist. Ziel der eingereichten Arbeit soll es sein, pflegerisches Handeln, Abläufe und Prozesse kritisch zu bewerten und zu zeigen, wie das Arbeitsumfeld und die Qualität der nephrologischen Pflege verbessert werden können.

Ein Gremium aus Gutachtern der AfnP und externen Spezialisten bewertet die Arbeiten. Überreicht wird der Pflegepreis im Rahmen des 30. AfnP-Symposiums in Fulda. Im Anschluss daran wird der prämierte Beitrag in der Zeitschrift Dialyse aktuell veröffentlicht. Die Arbeit senden Sie bitte an die Geschäftsstelle der AfnP. Dort erhalten Sie auch nähere Auskunft zu den Bewerbungs- und Bewertungskriterien des Pflegepreises.

\section{Das 30. AfnP-Symposium}

Ende Oktober feiern wir das 30. AfnPSymposium. Im großen Stadtsaal Fulda ist in den Pausen die Ausstellung „Geschichte der Nephrologie“ mit vielen technischen Geräten und weitreichenden Informationen geöffnet. Die Vorträge drehen sich diesmal um die Peritonealdialyse und die Heimhämodialyse sowie die nächtliche Zentrumsdialyse. Das Thema Mangelernährung und Ernährungsstatus finden Sie im Hauptprogramm und ergänzend in Workshops. Außerdem können Sie über Palliativmedizin, Patientenverfügung, Probleme älterer Patienten sowie langjährige, inzwischen ältere Mitarbeiter in Dialyseeinrichtungen diskutieren. Umsetzung und Einführung von QM sowie ein technisch orientiertes Parallelprogramm mit Themen aus den Bereichen Sicherheitsüberprüfung von Versorgungssystemen in Dialyseeinrichtungen und Aufbau und Funktion des Dialysators sind zwei weitere Eckpfeiler des Programms.

Wie immer greifen wir die wichtigen Gefäßzugänge mit drei Workshops von Prof. Gerhard Krönung, Ottweiler, und Vorträgen im Hauptprogramm (z. B. Katheterblocking, Gefäßanschluss nach der Hygieneleitlinie) auf. Es wird zudem einen Übersichtsvortrag zur Apheresetherapie geben - eine Behandlung, die zunehmend in Dialyseeinrichtungen angeboten wird. Nicole Scherhag, Saulheim, wird wie im letzten Jahr über Dialyse und Soziales mit dem diesjährigen Schwerpunkt Pflegeversicherung referieren. Weitere Themen sind Aktuelles zum Diabetes, Akutverfahren, Nephroprotektion, Immunsuppression nach Transplantation und vieles mehr. Selbstverständlich können Sie wieder ärztliche und pflegerische Fortbildungspunkte für diese Veranstaltung sammeln. Das aktuelle Programm und die Kurzfassung finden sie auf unserer Homepage.

Auf Wiedersehen in Fulda!

Ihre Marion Bundschu 